\title{
Modeling Student Relationship in Class: A Social Network Evaluation
}

\author{
HUANG Sui ${ }^{1,}$,, ZHAN Donghua ${ }^{2, b}$ \\ ${ }^{1}$ Department of Computer Science, Jinan University, PR China \\ ${ }^{2}$ Xiangzhou Experimental School of Zhuhai, PR China \\ aths@jnu.edu.cn, ${ }^{b}$ dhzhan126@126.com
}

Keywords: relationship modeling, social network evaluation, peer group, correlation analysis.

Abstract. Relationship status contributes uniquely to student's emotional adjustment and academic performance. We use a half anonymous survey tool to establish favorite and exclusionary social relationship in class and aim to develop a model to evaluate holistic relationship status personally. Social network centrality features and peer group factors are selected for evaluation modeling. Correlation analysis is conducted to confirm the independency and validity of the evaluation method. 254 students participated in our study and provided 1523 favorite ties and 891 exclusionary ties. Results show our method effectively reveals the student relationship, objectively considers the ties impact and promises to be a novel evaluation scale for every student in class.

\section{Introduction}

According to current Chinese educational system, most students will be organized in fixed classes for a long time, especially during 6-year primary school stage. Studies have demonstrated the profound impact of relationship between students in class ${ }^{[1,2]}$. We summarize such relationship with three kinds of characteristics: stable, implicit and personal. A variety of methods have been exploited to obtain student social relationship data, including many kinds of questionnaires, survey instrument and even personal interview ${ }^{[3]}$. But these methods have overt defect: the inadequate of privacy protection or the lost of individual link. Therefore, how to discover such covert ties truthfully and completely, and how to evaluate the relationship status objectively for every student, become the focus of our research interest. To issue these problems, we firstly introduced a half anonymous survey tool ${ }^{[4]}$ to collect social relationship data and establish favorite and exclusionary social network in class level; secondly selected in-degree, centrality, medium features and tripartite group factors to build evaluation model; finally, to verify the objectiveness and fairness of the model, we calculated the correlation coefficient with student's academic outcome.

\section{Method}

\section{Data Acquisition}

Although literature reviews have proposed some methods to collect students' social relationship data ${ }^{[5]}$, the real-name trait of the questionnaire or interview derogates from the veracity and integrality of survey results and the indirect survey instruments also suffer from the same problems. To issue this problem, we introduced a half anonymous survey instrument. Reference [4] demonstrated the principle and process of this method. 254 students (148 male, 106 female) from grade 4 to 6 in five classes participated in our survey and provided 1523 favorite ties and 891 exclusion ties. For each class, two social networks (the favorite network and exclusionary network) are established based on above data. Fig. 1 is a represent favorite network drawn by social network analysis tool Pajek ${ }^{[6]}$. Where yellow nodes represent male and blue nodes stand for female. The nodes will have the size of shape proportionate to the votes received. Despite the pattern and distribution differences of relationship networks, general properties, such as more favorite ties than exclusionary ties, larger peer group among male students and less ties between opposite sex are confirmed by all networks. 


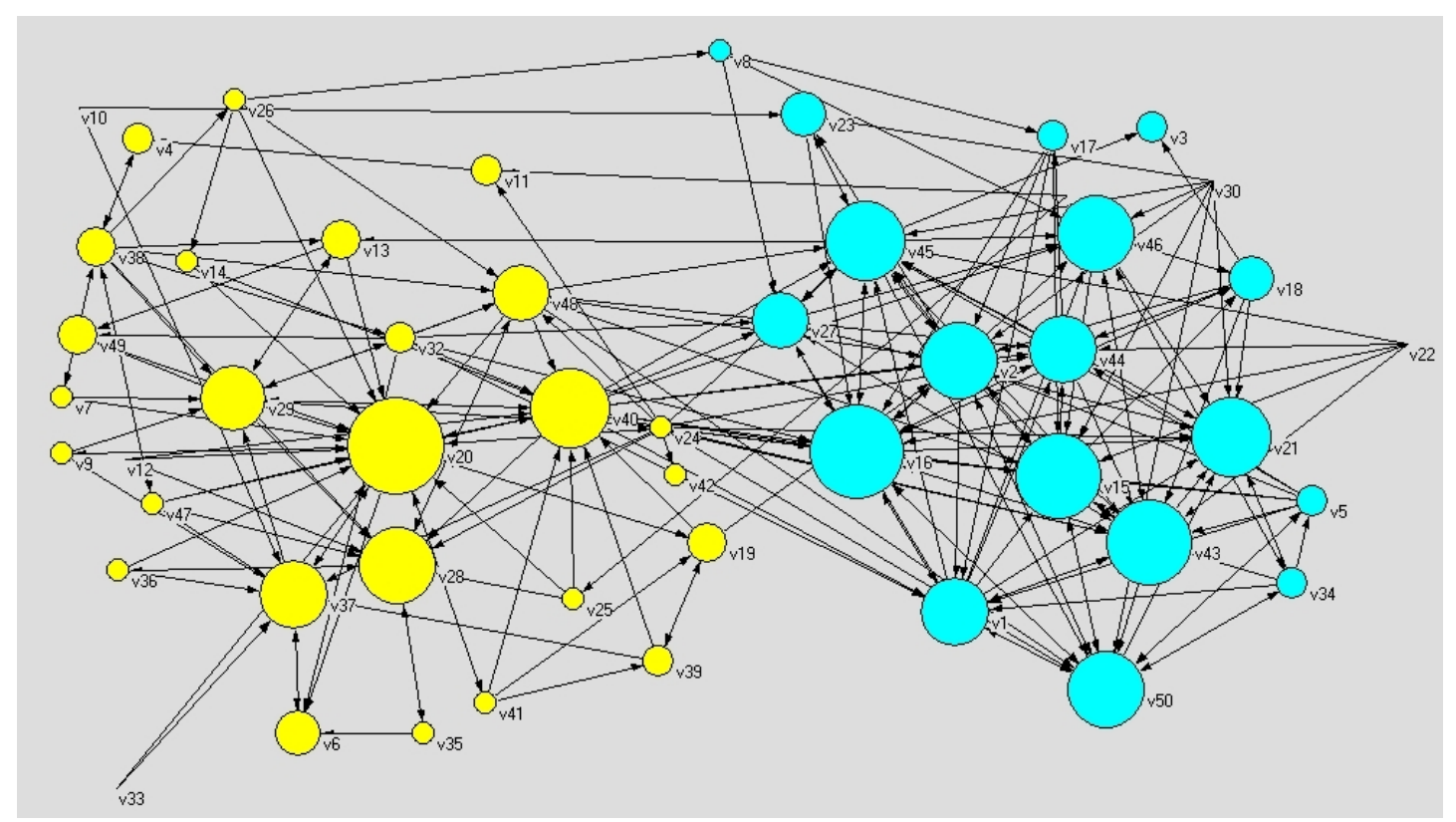

Figure 1. The favorite relationship network in a class

\section{Modeling Method}

The focus of our study is modeling the social relationship status in class. Student's favorite and exclusionary network provide us with rich assessment stuff. Since the obvious semantic difference of the two networks, the network features ought to treat synthetically. In addition, the standard class size in Chinese primary school is 50 . Therefore, many community analysis algorithms are not suited to this context. For simplicity and practicality, we select in-degree, centrality and medium features as node's basic characteristics and 13 trilateral peer group configurations as community trait. These factors are weighted and integrated to build our assessment model. The evaluation grade for each student is composed of two aspects: positive grade from favorite network and negative grade from exclusionary network. Firstly, the favorite or exclusionary degree is counted by in-degree feature for every node. Secondly, the centrality and medium feature is calculated. Thirdly, 13 tripartite combinations are considered. If a node arises in the above configurations, it will get a base score increment. But different configuration or different role in the configuration will get different weight for their special semantic value. For example, nodes in configuration 16-300 will obtain the highest coefficient award and the medium node in 5-021U and 5-021D will be endowed higher weight. Finally, all the numbers are summed and normalized. Table. 1 shows the overall evaluation results of the five classes base on the favorite relationship networks.

Table 1. The overall favorite network evaluation results of five classes

\begin{tabular}{|c|c|c|c|c|c|c|}
\hline Class & $\begin{array}{c}\text { Average } \\
\text { in-degree }\end{array}$ & $\begin{array}{c}\text { In-degree } \\
\text { STDEV }\end{array}$ & $\begin{array}{c}\text { Avg. medium } \\
\text { score }\end{array}$ & $\begin{array}{c}\text { Medium } \\
\text { STDEV }\end{array}$ & $\begin{array}{c}\text { tripartite } \\
\text { Avg. score }\end{array}$ & $\begin{array}{c}\text { Tripartite } \\
\text { STDEV }\end{array}$ \\
\hline 1 & 4.306 & 4.5289 & 0.04385 & 0.07402 & 0.78 & 0.72241 \\
\hline 2 & 5.137 & 3.7086 & 0.03323 & 0.04152 & 0.79 & 0.6103 \\
\hline 3 & 3.946 & 3.4082 & 0.02919 & 0.03175 & 0.73 & 0.7821 \\
\hline 4 & 6.105 & 6.0456 & 0.06671 & 0.07821 & 0.81 & 0.8261 \\
\hline 5 & 4.872 & 4.6357 & 0.04787 & 0.07533 & 0.76 & 0.7559 \\
\hline
\end{tabular}

\section{Correlation Analysis}

We expect social relationship score to be an independent and impactful index to evaluate the influence in the emotion, character and study of the student. To approve this hypothesis, both academic and social network scores about individuals are needed. A suite of authenticated transcript records on Chinese, English and Mathematics from a class are employed to conduct the verification. Records are 
normalized firstly, then calculated in Z-score. Table 2 shows the Pearson test results of the verification. In Table 2, F. ratio and E. ratio indicate Favorite and exclusionary score weight respectively. Binary mixed stands for binary network and mixed study score of the three lessons. In binary network, only ballot is counted in despite of the vote order. While in multi-value network, we count ballot as well as order coefficient. Fig. 2 shows the detailed results of the test. Results show that there is a statistically appropriate correlation between student's social relationship score and academic score. The direction of the correlation is positive, which means that students obtaining high relationship scores tended to obtain high academic scores.

Table 2. The Pearson test results between network score and mixed study outcome

\begin{tabular}{|c|c|c|c|}
\hline F. ratio & E. ratio & Binary mixed & Multivalue mixed \\
\hline 0.9 & 0.1 & 0.5817 & 0.5788 \\
\hline 0.8 & 0.2 & 0.5648 & 0.5616 \\
\hline 0.7 & 0.3 & 0.5349 & 0.5314 \\
\hline 0.6 & 0.4 & 0.4910 & 0.4870 \\
\hline 0.5 & 0.5 & 0.4347 & 0.4302 \\
\hline
\end{tabular}
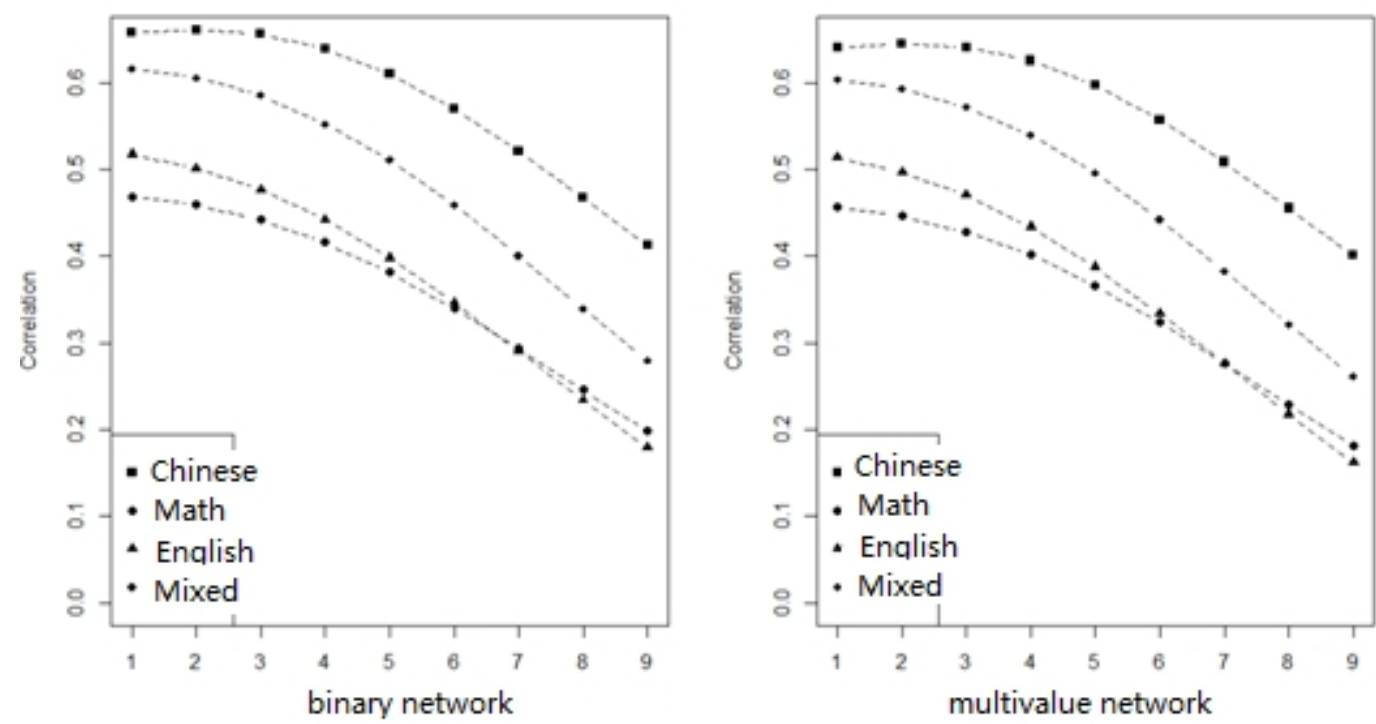

Figure 2. The Pearson test results between academic scores and relationship scores

\section{Conclusions}

Social relationship status is critical when evaluating the circumstances of students in class. Existing methods have shortcomings in relationship data acquisition and assessment modeling. We proposed two ways to issue the problem. First, we introduced a special survey tool to obtain social relationship data in class and establish favorite and exclusionary network. Second, we developed a model to evaluate the relationship status with network centrality features and peer group factors synthetically. In addition, to verify the independency and significance of the model, we conducted a Pearson test between relationship scores and academic scores. Experimental results showed that our integrated method established a new scale to assess the status of social relationship for student. Such scale is statistically moderate correlation with academic outcome and is provided with good independency and validity. In general, this work is one of the steps of our social relationship research project on improving the administration of primary school class and boosting the students' harmonious relationship. 


\section{References}

[1] Tuire Palonen, Marita Neitola: Friendship ties and exclusionary ties in classroom. The social structure among eight years old children, Proceedings of the 8th international conference on International conference for the learning sciences - Vol.2 (2008), p.164-171

[2] A. Martinez, Y. Dimitriadis, B. Rubia, E. Gomez, P.de la Fuente: Combining qualitative evaluation and social network analysis for the study of classroom social interactions, Computers \& Education Vol.41 (2003), p.353-368.

[3] Chloe Brown1, Neal Lathia1, Cecilia Mascolo1, Anastasios Noulas1,Vincent Blondel: Group Colocation Behavior in Technological Social Networks, PloS one, Vol.9, Issue 8(2014), p.e105816

[4] Huang Sui, Zhan Donghua: Empirical Research on Class Environment Evaluation, 4th International Conference on Computer Science and Network Technology. Vol. 1(2015), p. $225-228$

[5] Xin Jin, Chi Wang, Jiebo Luo: LikeMiner: A System for Mining the Power of 'Like' in Social Media Networks. KDD'11, August 21-24, 2011, pp. 753-756

[6] Wouter de Nooy, Andrej Mrvar, Vladimir Batagelj: Exploratory Social Network Analysis with Pajek, Cambridge University Press and World Publishing Corporation (2012) 